\title{
Participación materna en prevención y control de anemia con micronutrientes en lactantes. Distrito de Independencia, Lima - 2015
}

Lorena Lozano-Villafuerte* 1,a; Luzmila Troncoso-Corzo ${ }^{2, b}$; Víctor Noriega-Ruiz 2,3,c

RESUMEN

Objetivo: Identificar el nivel de participación materna en el cumplimiento del esquema de suplementación con micronutrientes (MN) para la prevención y control de anemia en niños menores de 24 meses en el distrito de Independencia en Lima entre el 2015 y 2016.

Materiales y métodos: Estudio descriptivo, observacional, longitudinal, retrospectivo realizado en el centro de salud Ermitaño Bajo. Participaron madres cuyos niños iniciaron suplementación con micronutrientes. Los datos se recolectaron de 40 historias clínicas seleccionadas. El análisis de datos se realizó mediante estadísticas descriptivas. Principales medidas de resultados: I) Participación alta: recibió $\geq 181$ sobres (MN) y realizó $\geq 2$ dosajes de hemoglobina al niño. II) Participación intermedia alta: recibió $\geq 181$ sobres (MN) y realizó $\leq 1$ dosaje de hemoglobina. III) Participación intermedia baja: recibió $\leq 180$ sobres (MN) y realizó $\geq 2$ dosajes de hemoglobina. IV) Participación baja: recibió $\leq 180$ sobres (MN) y realizó $\leq 1$ dosaje de hemoglobina.

Resultados: I) Participación alta: 9 (22,5\%), II) Participación intermedia alta: 3 (7,5 \%), III) Participación intermedia baja: 5 (12 5 \%) y IV) Participación baja: 23 (57,5\%).

Conclusiones: Solo 9 madres $(22,5 \%)$ tuvieron una participación alta en el cumplimiento del esquema de suplementación con micronutrientes para prevenir y controlar la anemia en niños menores de 24 meses en el Centro de Salud Ermitaño Bajo del distrito de Independencia.

Palabras clave: Anemia; Conducta materna; Alimentación suplementaria; Micronutrientes (Fuente: DeCS BIREME).

\section{Maternal engagement in infant's prevention and control of anemia with micronutrients. District of Independencia, Lima - 2015}

\section{ABSTRACT}

Objective: To identify the level of maternal engagement in complying with the micronutrient (MN) supplementation guidelines for the prevention and control of anemia in children under 24 months of age "in the district of Independencia, Lima, between the years 2015 and 2016".

Materials and methods: A descriptive, observational, longitudinal and retrospective study was performed at the Centro de Salud Ermitaño Bajo, district of Independencia, Lima, between the years 2015 and 2016. Participants were mothers whose children started micronutrient supplementation. Data was collected from 40 selected medical records. Data analysis was performed using descriptive statistics. Main outcome measures were: I) High level of engagement: the infant received $\geq 181$ $M N$ sachets and $\geq 2$ hemoglobin doses. II) Intermediate-high level of engagement: the infant received $\geq 181 \mathrm{MN}$ sachets and $\leq 1$ hemoglobin dose. III) Intermediate-low level of engagement: the infant received $\leq 180 \mathrm{MN}$ sachets and $\geq 2$ hemoglobin doses. IV) Low level of engagement: the infant received $\leq 180 \mathrm{MN}$ sachets and $\leq 1$ hemoglobin dose.

Results: I) High level of engagement: 9 mothers (22.5\%). II) Intermediate-high level of engagement: 3 mothers (7.5\%). III) Intermediate-low level of engagement: 5 mothers (12.5\%). IV) Low level of engagement: 23 mothers (57.5\%).

Conclusions: Only 9 (22.5\%) mothers had a high level of engagement in complying with the micronutrient supplementation guidelinesfor the prevention and control of anemia in children under 24 months of age at the Centro de Salud Ermitaño Bajo, district of Independencia.

Keywords: Anemia; Maternal behavior; Supplementary feeding; Micronutrients (Source: MeSH NLM).

1. Centro de Salud Ermitaño Bajo. Lima, Perú.

2. Universidad Nacional Mayor de San Marcos, Facultad de Medicina. Lima, Perú.

3. Hospital Cayetano Heredia. Lima, Perú.

a. Licenciada en nutrición, Maestrando de la Maestría en Nutrición Clínica.

b. Médico Cirujano, Doctora en Medicina, Docente investigador.

c. Médico Endocrinólogo, Maestrando de la Maestría en Nutrición Clínica.

* Autor corresponsal. 


\section{INTRODUCCIÓN}

Según la Organización Mundial de la Salud, la anemia "es un trastorno en el cual el número de eritrocitos (y, por consiguiente, la capacidad de transporte de oxígeno de la sangre) es insuficiente para satisfacer las necesidades del organismo" (1). En el 2011, se estimó que la anemia afectaba a alrededor de 800 millones de niños y mujeres en el mundo. De los cuales, 528,7 millones eran mujeres gestantes o en edad reproductiva; y 273,2 millones eran niños menores de 5 años, y aproximadamente la mitad de ellos también son deficientes de hierro ${ }^{(2)}$. En Latinoamérica, la anemia por deficiencia de hierro afecta cerca del $50 \%$ de los niños menores de dos años ${ }^{(3)}$.

En el Perú existe una alta prevalencia nacional de anemia, cuatro de cada diez niños menores de tres años de edad (44,4 \%) padecen de esta enfermedad. En la provincia de Lima alcanza el 35,5\%; sin embargo, los más afectados son los menores de 18 meses, que superan el $59 \%$ (4). Se conoce que la anemia en la infancia genera consecuencias a largo plazo, afecta el desempeño cognitivo, lo cual repercutirá en la adquisición de capacidades desde los primeros años hasta la vida adulta. Dado este comportamiento epidemiológico de la anemia en los niños peruanos, se han generado iniciativas de salud pública implementadas en las últimas décadas para hacer frente a la enorme carga que la anemia le significa a la población infantil ${ }^{(5)}$.

En el 2012, la OMS, luego de una revisión sistemática de 8 estudios en distintos países, encontró que intervenciones con micronutrientes en polvo ayudaron a reducir la anemia, por lo cual ha emitido una directriz donde se ofrecen recomendaciones de ámbito mundial sobre el uso de micronutrientes en polvo para la fortificación domiciliaria de los alimentos consumidos por lactantes y niños pequeños de 6 a 23 meses de edad (6).

En el Perú, año 2014, se aprobó la Directiva Sanitaria № 056 que establecía la suplementación con multimicronutrientes y hierro para la prevención de anemia en menores de 36 meses, en nacidos a término (mayor de 37 semanas) y con peso igual o mayor a 2500 gramos. la suplementación fue diaria con 1 sobre de multimicronutrientes por día a partir de los 6 meses durante 12 meses continuos (360 sobres en total). Para la prevención y control de la anemia es necesario la determinación de la hemoglobina al inicio, a los 6 meses de iniciada la suplementación y al término de la misma (a los 12 meses) ${ }^{(7,8)}$. En adelante denominaremos al multimicronutriente como micronutriente, según su definición más reciente, al referirnos al producto que, como una mezcla de vitaminas y minerales que ayudan a prevenir la anemia y otras enfermedades, está compuesto por el hierro, zinc, ácido fólico, vitamina A y vitamina $\mathrm{C}^{(9)}$.

El objetivo del presente estudio fue identificar el nivel de participación materna en el cumplimiento del esquema de suplementación con micronutrientes para la prevención y control de anemia en niños menores de 24 meses en un centro de salud de Independencia en Lima durante el año 2015 y 2016.

\section{MATERIALES Y MÉTODOS}

\section{Diseño y población de estudio}

Se realizó un estudio de tipo descriptivo, observacional, longitudinal y retrospectivo, desde abril de 2015 hasta junio de 2016 en el centro de salud Ermitaño Bajo del distrito de Independencia, ubicado en el departamento de Lima, Perú. Establecimiento de salud del primer nivel de atención con un estimado de 425 familias asignadas. El estudio fue aprobado por la Unidad de Capacitación de la ex-Red de Salud Túpac Amaru.

Se revisó el archivo de registros e historias clínicas de todos los niños atendidos desde abril 2015 en el Control de Crecimiento y Desarrollo, y cuyas madres empezaron a recibir los micronutrientes para la suplementación preventiva de anemia.

Los criterios de inclusión fueron historias clínicas de niños entre 6 a 9 meses de edad, aparentemente sanos, y que habían iniciado la suplementación con micronutrientes. Criterio de exclusión: Niños que recibían otro tipo de suplemento.

\section{Variables y mediciones}

La información fue recolectada en una ficha de datos elaborada para este estudio, incluía información del niño, registro de administración de micronutrientes, dosajes de hemoglobina y datos de la madre.

Las variables analizadas para valorar la participación materna fueron la cantidad de sobres de micronutrientes recibidos (se consideró que el niño debe recibir 30 sobres cada mes, durante 12 meses, en total, 360 sobres), según esquema vigente; y la realización de dosajes de hemoglobina (valor menor a $11 \mathrm{~g} / \mathrm{dL}$ se consideró anemia) ${ }^{(9)}$.

Para la elaboración de la escala de participación materna se consideró la acción voluntaria de la madre que acude a recibir micronutrientes y realiza los dosajes de hemoglobina $(\mathrm{Hb})$ al niño. Para para identificar el nivel de participación se planteó el cumplimiento mayor al 50 \% del esquema de suplementación con micronutrientes (MN) propuesto por el MINSA; para los 
niveles de participación intermedia alta y participación alta se consideró, además, la evidencia de que las intervenciones eficaces de prevención y control de anemia tienen un promedio de duración de 6 meses ${ }^{(6,9)}$.

Principales medidas de resultados:

I) Participación alta: recibió $\geq 181$ sobres $(M N)$ y realizó $\geq 2$ dosajes de hemoglobina.

II) Participación intermedia alta: recibió $\geq 181$ sobres (MN) y realizó $\leq 1$ dosaje de hemoglobina.

III) Participación intermedia baja: recibió $\leq 180$ sobres (MN) y realizó $\geq 2$ dosajes de hemoglobina.

IV) Participación baja: recibió $\leq 180$ sobres (MN) y realizó $\leq 1$ dosaje de hemoglobina.

\section{Análisis estadístico}

Los datos recogidos fueron ingresados a una base de datos en Excel, luego se aplicaron pruebas de estadística descriptiva, frecuencia y porcentaje con el programa estadístico SPSS (Statistical Package for the Social Science) versión 22.0, los datos se presentaron en tablas y para la elaboración de las figuras se empleó el software Microsoft Excel 2013.

\section{RESULTADOS}

Se seleccionó un total de 40 historias clínicas de niños que cumplían los criterios de inclusión. Respecto a las características del niño, la edad promedio de inicio de suplementación fue de 7,1 $\pm 1,1$ meses; $23(57,5 \%)$ fueron de sexo femenino y 17 (42,5\%), masculino. Respecto al estado nutricional del niño, 22 (55\%) presentaron crecimiento adecuado y 18 (45\%) crecimiento inadecuado, con evidencia de ganancia mínima o excesiva de peso y/o la ganancia mínima de talla, según criterios establecidos para la evaluación del crecimiento y estado nutricional ${ }^{(10)}$.

Suplementación con micronutrientes: El número promedio de sobres de $M N$ recibidos por niño fue de 138 sobres, con un rango de 30 a 360 sobres de $M N$. De los 40 casos, solo 1 (2,5 \%) culminó con el esquema de suplementación (recibió 360 sobres) (Figura 1).

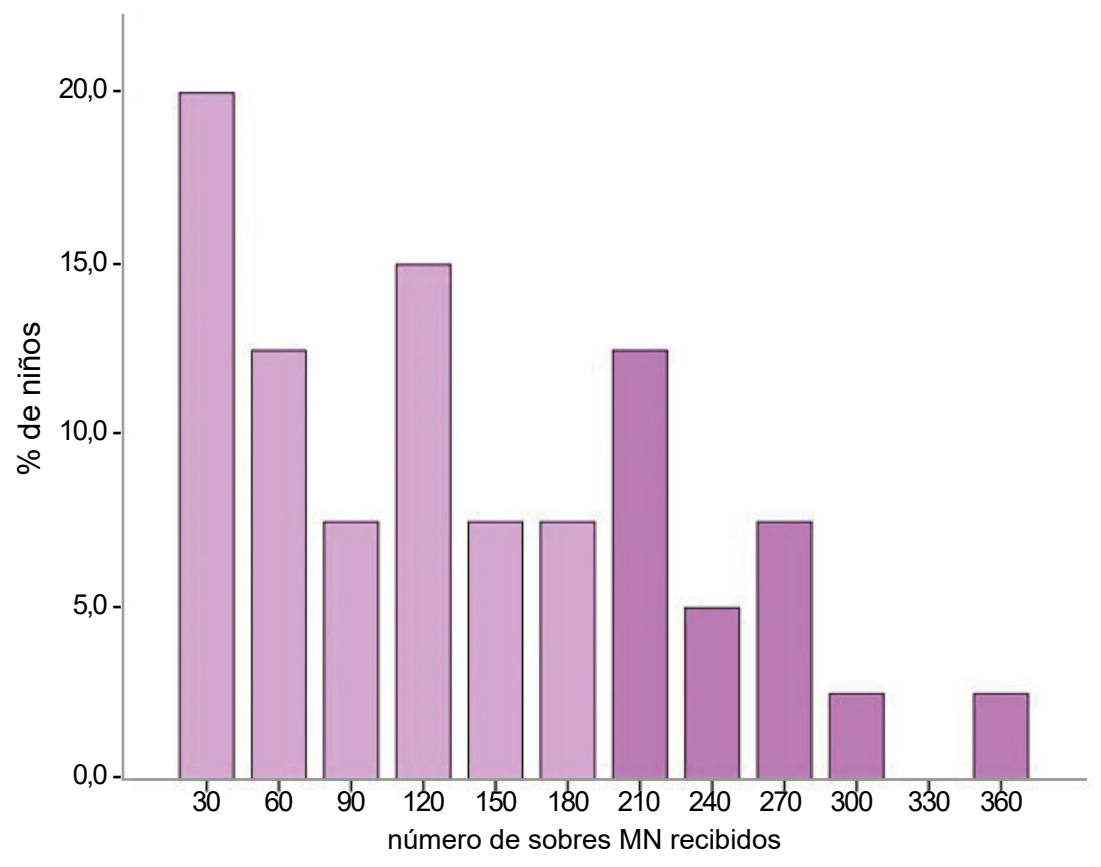

Figura 1. Cantidad de sobres de micronutrientes recibidos por la madre $(n=40)$

Dosaje de hemoglobina: Las madres que realizaron el primer dosaje de hemoglobina fueron 34 (85\%), la media de la concentración del primer dosaje de hemoglobina fue de $10,7 \pm 0,7 \mathrm{~g} / \mathrm{dl}$.; 14 madres (35\%) acudieron a realizar el segundo dosaje de $\mathrm{Hb}$, de los cuales se observó que 4 de ellos se lograron recuperar de la anemia inicial luego de haber recibido 180 sobres de MN o más (Figura 2). 


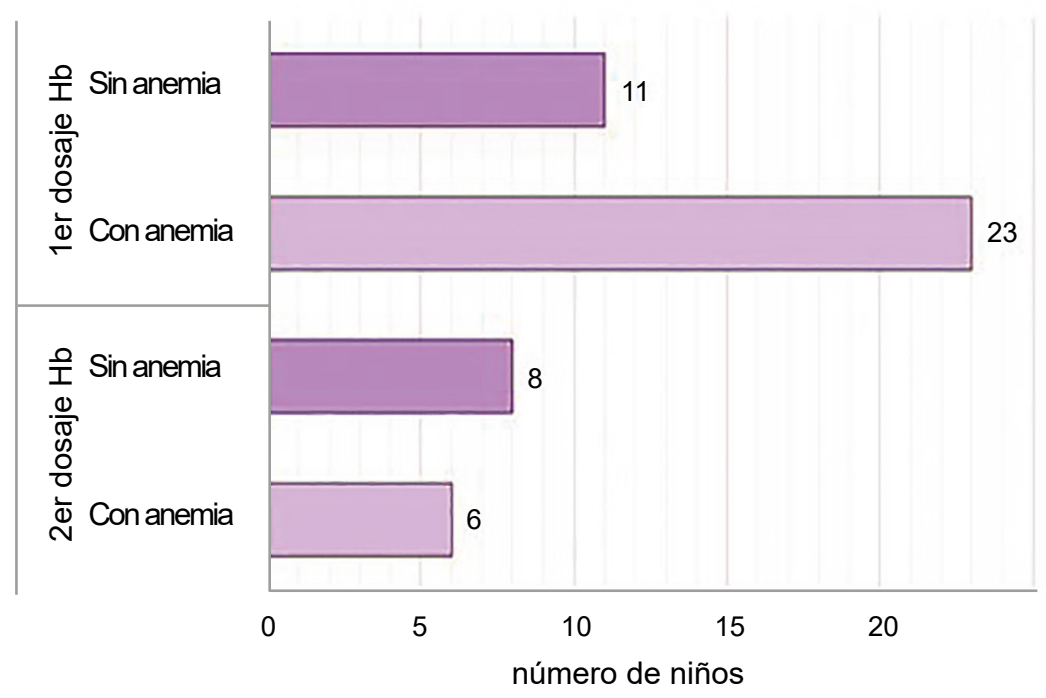

Figura 2. Dosajes y concentración de hemoglobina en los niños $(n=40)$

En relación al nivel de participación materna, de los 40 casos, solo 12 madres ( $30 \%$ ) acudieron al centro de salud a recibir más de 181 sobres de $M N$. Respecto al dosaje de hemoglobina, solo 14 madres (35\%) realizaron 2 o más dosajes de hemoglobina a sus niños (Tabla 1 ).

Tabla 1. Número de sobres de $M N$ recibidos y dosajes de hemoglobina realizados $(n=40)$

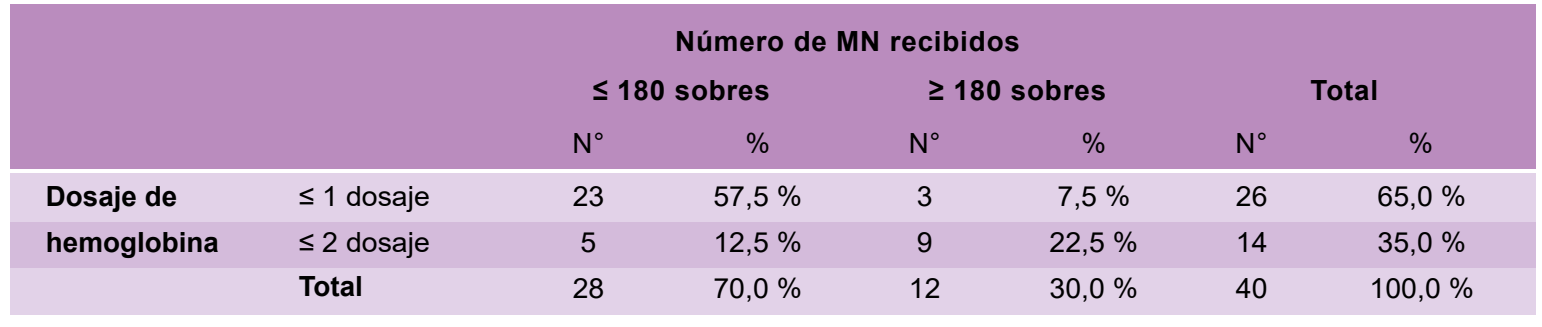

En cuanto a la participación materna, al considerar sobres recibidos y dosajes realizados, se encontró una participación alta en 9 casos $(22,5 \%)$, participación intermedia alta ( 3 madres que corresponden al $(7,5 \%)$, participación intermedia baja, 5 casos (12,5\%), y participación baja, 23 madres $(57,5 \%)$.

Respecto a las características de la madre, la edad promedio fue de 27,05 $\pm 7,3$ años, 16 (40\%) refirieron tener educación de nivel secundaria completa, y respecto al estado civil 27 (67,5\%) manifestaron ser convivientes. Asimismo, de acuerdo con las respuestas de las madres, en 8 ( $20 \%)$ de los casos, el padre del niño participa poco o no participa en el cuidado del mismo (Tabla 2). 
Tabla 2. Características de la madre y nivel de participación materna en el cumplimiento del esquema de suplementación con MN ( $\mathrm{n}=40$ )

\begin{tabular}{|c|c|c|c|c|c|c|c|c|c|}
\hline \multicolumn{10}{|c|}{ Nivel de participación materna } \\
\hline \multirow{2}{*}{\multicolumn{2}{|c|}{ Caracteristica de la madre }} & \multicolumn{2}{|c|}{$\begin{array}{l}\text { Participacíón } \\
\text { alta }\end{array}$} & \multicolumn{2}{|c|}{$\begin{array}{c}\text { Participacíón } \\
\text { intermedia } \\
\text { alta }\end{array}$} & \multicolumn{2}{|c|}{$\begin{array}{c}\text { Participacíón } \\
\text { intermedia } \\
\text { baja }\end{array}$} & \multicolumn{2}{|c|}{$\begin{array}{c}\text { Participacíón } \\
\text { baja }\end{array}$} \\
\hline & & $\mathrm{N}^{\circ}$ & $\%$ & $\mathrm{~N}^{\circ}$ & $\%$ & $\mathrm{~N}^{\circ}$ & $\%$ & $\mathrm{~N}^{\circ}$ & $\%$ \\
\hline \multirow{3}{*}{$\begin{array}{l}\text { Edad de la } \\
\text { madre }\end{array}$} & $<18$ años & & & & & & & 2 & $5,0 \%$ \\
\hline & 18 a 29 años & 7 & $17,5 \%$ & 2 & $5,0 \%$ & 1 & $2,5 \%$ & 11 & $27,5 \%$ \\
\hline & 30 a más años & 2 & $5,0 \%$ & 1 & $2,5 \%$ & 4 & $10,0 \%$ & 10 & $25,0 \%$ \\
\hline \multirow{6}{*}{$\begin{array}{l}\text { Grado de } \\
\text { instrucción }\end{array}$} & Primaria & & & & & & & 3 & $7,5 \%$ \\
\hline & $\begin{array}{l}\text { Secundaria } \\
\text { incompleta }\end{array}$ & 3 & $7,5 \%$ & 1 & $2,5 \%$ & 1 & $2,5 \%$ & 6 & $15,0 \%$ \\
\hline & $\begin{array}{l}\text { Secundaria } \\
\text { incompleta }\end{array}$ & 5 & $12,5 \%$ & 2 & $5,0 \%$ & 2 & $5,0 \%$ & 7 & $17,5 \%$ \\
\hline & $\begin{array}{l}\text { Superior no } \\
\text { universitario }\end{array}$ & 1 & $2,5 \%$ & & & 1 & $2,5 \%$ & 4 & $10,0 \%$ \\
\hline & $\begin{array}{l}\text { Superior } \\
\text { universitario }\end{array}$ & & & & & & & & \\
\hline & No registra & & & & & 1 & $2,5 \%$ & 3 & $7,5 \%$ \\
\hline \multirow[t]{3}{*}{ Estado civil } & Soltera & 2 & $5,0 \%$ & & & & & 2 & $5,0 \%$ \\
\hline & Casada & 2 & $5,0 \%$ & 1 & $2,5 \%$ & & & 2 & $5,0 \%$ \\
\hline & Conviviente & 5 & $12,5 \%$ & 2 & $5,0 \%$ & 4 & $10,0 \%$ & 16 & $40,0 \%$ \\
\hline
\end{tabular}

Las limitaciones con respecto a las características de las madres, fueron registradas en el formato de atención integral del niño presente en la historia clínica; sin embargo, algunos formatos se encontraron con descripciones incompletas.

\section{DISCUSIÓN}

Respecto a la suplementación con $\mathrm{MN}$, de los 40 casos, $8(20 \%)$ recibieron $M N$ en el control inicial ( 30 sobres) y no retornaron por las siguientes dosis; 12 (30\%) lograron recibir más de la mitad del esquema de suplementación recomendado (181 sobres a más), solo 1 madre $(2,5 \%)$ acudió a recibir el esquema completo de suplementación (360 sobres) en el tiempo esperado, lo cual indicaría que no solo se trata de proveer el producto a la madre, sino sensibilizarla sobre la importancia de fortificar los alimentos con micronutrientes. Según un ensayo comunitario desarrollado en cuatro regiones del país por el Instituto Nacional de Salud, existen barreras que afectan la adherencia a la suplementación con $M N$, como por ejemplo, que las madres desconocen las consecuencias de la anemia y/o como prevenirla, por lo cual no consideran que el micronutriente tenga un beneficio tangible o inmediato; además, mencionaron que altera el sabor del alimento y provoca rechazo, que no tenían tiempo para acudir al centro de salud o se olvidaban de usarlo ${ }^{(11)}$.

En relación al resultado de hemoglobina del primer dosaje, $23(67,6 \%)$ presentaron algún grado de anemia, de ellos, solo 14 madres llevaron a sus niños a realizar el segundo dosaje de $\mathrm{Hb}$ para el seguimiento de la anemia, lo cual podría evidenciar la poca información de las madres respecto a los efectos negativos de la anemia en el niño. Junco J, en el 2015 reportó con base en una encuesta realizada a madres de niños beneficiarios del programa de suplementación con $M N$ en Ayacucho, que el 31 \% de ellas refirió que no le explicaron respecto a la importancia de este análisis, y al $24 \%$ no le informaron sobre el resultado del dosaje, esa situación podría ser una limitante por la que la madre no tome interés en los controles de hemoglobina ${ }^{(12)}$.

En cuanto a la recuperación de la anemia, en el segundo dosaje 4 niños que presentaron el cuadro al inicio lograron mejorar la concentración de $\mathrm{Hb}$ y alcanzaron niveles normales, sus madres, además, habían recibido 
180 sobres de $M N$ o más. La efectividad de los $M N$ ha sido corroborada en distintos estudios; Munayco et al. realizaron un estudio en niños de 6 a 35 meses de edad, de 29 establecimientos de Andahuaylas, Ayacucho y Huancavelica, a quienes se les indicó $M N$ por un periodo de 12 meses. De 1325 niños, solo 759 culminaron la suplementación, la prevalencia de anemia se redujo de 70,2 a $36,6 \%(p<0,01)$, la media de Hb se incrementó en $0,8 \mathrm{~g} / \mathrm{dL}$ de manera global ${ }^{(13,14)}$.

Por otro lado, en 4 casos que presentaron anemia según el primer dosaje, no lograron mejorar la concentración de $\mathrm{Hb}$ aunque sus madres recibieron 150 sobres de $M N$ a más. Huaman-Espino et al., en un estudio de corte transversal, evaluaron la cantidad y calidad de sobres de $M N$ consumidos, y se encontró menor prevalencia de anemia en aquellos niños que consumían el suplemento en forma adecuada, ya que no basta con consumir la cantidad necesaria de $\mathrm{MN}$, sino asegurar que la forma de preparación y el proceso de consumo sea el correcto (15). Sin embargo, la anemia podría estar relacionada a otras causas, como parasitosis o al déficit de nutrientes diferentes al hierro como vitamina B12, ácido fólico, vitamina $A$ y cobre, entre otros ${ }^{(16)}$.

En conclusión, de las 9 madres (22,5\%) que tuvieron una participación alta en el cumplimiento del esquema de suplementación, la mayoría fueron jóvenes, 7 de ellas menores de 30 años; además, se identificó una participación baja en 23 madres $(57,5 \%)$, en esta categoría se encuentran las 2 madres adolescentes del grupo y aquellas que refirieron tener nivel de educación primaria. Algunos datos de las madres no fueron registrados, lo cual no permite caracterizarlas o considerar su nivel educativo, dato que debería valorar el personal de salud para emplear una comunicación más asertiva y efectiva ${ }^{(12)}$.

El personal de salud es corresponsable en el proceso de suplementación con $M N$, es necesario fortalecer las habilidades en la consejería nutricional y no solo centrarse en el "cómo" preparar y dar los MN, si no que los cuidadores necesitan conocer el "porqué" de la suplementación (17). Es necesario optimizar la comunicación educativa hacia la madre, para motivarla y lograr la sostenibilidad en el tiempo de la suplementación, hacer uso de material educativo, realizar sesiones demostrativas, visitas domiciliarias para mejorar adherencia, capacitar e incorporar agentes y líderes comunitarios en el acompañamiento y seguimiento del consumo de $M N{ }^{(11)}$.

Además, es necesario incluir temas de cuidado de la salud, nutrición en la adolescencia y embarazo, lactancia materna, entre otros, desde el nivel de educación primaria y secundaria, para mejorar el estado nutricional de nuestras adolescentes y mujeres en edad fértil, ya que existe evidencia de que las intervenciones eficaces para abordar la carencia de micronutrientes se dan en la fase pregestacional y gestacional, meta mundial establecida en la última Asamblea Mundial de la Salud ${ }^{(18)}$.

Agradecimiento: A los coautores, Luzmila Troncoso Corzo y Víctor Noriega Ruiz por el soporte técnico, a la Red de Salud Túpac Amaru, a la Lic. Nut. Patricia Brito Saavedra y Lic. Nut. Mérida Santos Chafalote, coordinadora de la Estrategia Sanitaria de Alimentación y Nutrición Saludable de la RSTA y coordinadora de la ESANS de Microred Tahuantinsuyo respectivamente, por permitir la presente investigación y su estímulo al trabajo.

Contribuciones de autoría: 1a.Concepción y diseño del trabajo, recolección, análisis e interpretación de datos. 2 Análisis e interpretación de datos, revisión crítica del manuscrito. 3a Asesoría técnica y estadística.

\section{REFERENCIAS BIBLIOGRÁFICAS}

1. Organización Mundial de la Salud. Concentraciones de hemoglobina para diagnosticar la anemia y evaluar su gravedad. Ginebra: OMS [internet]; 2011. Disponible en: http://www.who.int/vmnis/indicators/haemoglobin_es.pdf

2. World Health Organization. The global prevalence of anemia in 2011. Geneva: WHO [internet]; 2015. Disponible en: http://www.who.int/nutrition/publications/micronutrients/ global_prevalence_anaemia_2011/en/. 2015 [citado 1 de julio de 2018].

3. AWGLA: Anemia Working Group Latin America. Compendio de guías latinoamericanas para el manejo de la anemia ferropénica. 2a ed. 2007.

4. Instituto Nacional de Estadística e Informática. Indicadores de resultados de los programas presupuestales, primer semestre 2017. Encuesta demográfica y de salud familiar. Lima: INEI [internet]; 2017. Disponible en: https://proyectos.inei.gob. pe/endes/images/Indicadores_Resultados_PPR_Primer_ Semestre_2017 [citado 1 de julio de 2018].pdf

5. Instituto Nacional de Salud. Anemia en la población infantil del Perú: Aspectos clave para su afronte. Lima: INS [Internet]; 2015. Disponible en: http://www.ins.gob.pe/repositorioaps/0/4/ jer/evidencias/ANEMIA FINAL_v.03mayo2015 [citado 1 de julio de 2018]..pdf.

6. Organización Mundial de la Salud. Directriz: Uso de micronutrientes en polvo para la fortificación domiciliaria de los alimentos consumidos por lactantes y niños de 6 a 23 meses de edad. Ginebra, OMS, 2012. Disponible en: http: / /apps. who. int/iris/bitstream/handle/10665/101016/9789243502045_ spa.pdf?sequence=1 2012 [citado 1 de julio de 2018].

7. Ministerio de Salud. Dirección General de Salud de las 
Personas. Directiva Sanitaria que establece la suplementación con Multimicronutrientes y hierro para la prevención de anemia en niñas y niños menores de 36 meses. Directiva Sanitaria $\mathrm{N}^{\circ}$ 056. Lima: MINSA [Internet]; [citado 1 de julio de 2018]. Disponible en: http://www.minsa.gob.pe/portada/ especiales/2015/nutriwawa/directivas/001DS_Suplem_ MultiMicro.pdf

8. Ministerio de Salud. Guía de práctica clínica para el diagnóstico y tratamiento de la anemia por deficiencia de hierro en niñas, niños y adolescentes en establecimiento de salud del primer nivel de atención. $\mathrm{RM} \mathrm{N}^{\circ}$ 028-2015. Lima: MINSA [Internet]; 2016. Disponible en: http://bvs.minsa.gob. pe/local/MINSA/3932. 2016 [citado 1 de julio de 2018].pdf

9. Ministerio de Salud. Dirección General de Salud de las Personas. Directiva Sanitaria para la prevención de anemia mediante la suplementación con micronutrientes y hierro en niñas y niños menores de 36 meses. Directiva Sanitaria N 068 . Lima: MINSA [Internet]; 2016. Disponible en: http:// bvs.minsa.gob.pe/local/MINSA/39312016 [citado 1 de julio de 2018].pdf

10. Ministerio de Salud. Norma Técnica de Salud para el Control de Crecimiento y Desarrollo de la Niña y el Niño Menor de Cinco Años. Dirección General de salud de las Personas. Lima: MINSA [Internet]; 2011. Disponible en: https://www.unicef. org/peru/spanish/NORMA_CRED. 2011 [citado 1 de julio de 2018]. pdf

11. Instituto Nacional de Salud. Centro Nacional de Alimentación y Nutrición. Reporte del Ensayo comunitario para mejorar la adherencia a la suplementación en niños de 6 a 35 meses de edad en el ámbito de las DIRESA/DISA Puno, Tacna, Loreto y Lima Este [Internet]. 2016. Disponible en: http: / / www. portal.ins.gob.pe/en/component/rsfiles / preview?path=cenan\%252FPrevencion\%2Bde\%2BRiesgo\%2By\%2 Bdano\%2Bnutricional\%252FReportes\%2Be\%2BInformes\%252FE nsayo_Comunitario. 2016 [citado 1 de noviembre de 2018]. pdf

12. Junco JE. Identificación de los factores que contribuyen y limitan la efectividad del programa de suplementación con multimicronutrientes en la reducción de la anemia de niños menores de tres años del ámbito rural de Vinchos de Ayacucho [Tesis para optar el grado de magister en gerencia social]. Lima, Perú: Pontificia Universidad Católica Del Perú, 2015. Disponible en: http://tesis.pucp.edu.pe/ repositorio/ 2015 [citado 1 de julio de 2018]. bitstream/ handle/123456789/6650/JUNCO_GUILLERMO_JORGE_ IDENTIFICACION_FACTORES.pdf? sequence $=1$

13. Munayco CV, Ulloa-Rea ME, Medina-Osis J, Lozano-Revollar CR, Tejada V, Castro-Salazar C, et al. Evaluación del impacto de los multimicronutrientes en polvo sobre la anemia infantil en tres regiones andinas del Perú. Rev Peru Med Exp Salud Pública. 2013;30(2):229-34.

14. Medina JL, Meza AM, Roque J. Eficacia del programa educativo

supervisado en la administración de multimicronutrientes para prevenir la anemia ferropénica en niños de 2 a 3 años en centros de estimulación Surco. Revista Científica Alas Peruanas.2014;1(2):1-29

15. Huamán-Espino L, Aparco JP, Núñez-Robles E, Gonzáles E, Pillaca J, Mayta-Tristán P. Consumo de suplementos con multimicronutrientes chispitas ${ }^{\circledR}$ y anemia en niños de 6 a 35 meses: estudio transversal en el contexto de una intervención poblacional en Apurímac, Perú. Rev Peru Med Exp Salud Pública. 2012;29(3):314-23.

16. Gonzales E, Huamán-Espino L, Gutiérrez C, Aparco JP, Pillaca J. Caracterización de la anemia en niños menores de cinco años de zonas urbanas de Huancavelica y Ucayali en el Perú. Rev Peru Med Exp Salud Pública. 2015;32(3):431-9.

17. Creed-Kanashiro H, Bartolini R, Abad M, Arévalo V. Promoting multi-micronutrient powders (MNP) in Perú: acceptance by caregivers and role of health personnel. Matern Child Nutr. 2016;12(1):152-63.

18. The Lancet. Serie 2013 sobre nutrición materno-infantil. Resumen Ejecutivo de la Serie sobre Nutrición Materno Infantil de The Lancet [Internet], 2013. Disponible en: http://www. incap.org.gt/index. 2013 [citado 1 de julio de 2018]. php/ es/publicaciones/doc_view/695-serie-2013-sobre-nutricionmaterno-infantil.

Fuentes de financiamiento:

Este artículo ha sido financiado por los autores.

Conflictos de interés:

Los autores declaran no tener ningún conflicto de interés.

\section{Correspondencia:}

Lorena Lois Lozano Villafuerte

Dirección: Jr. Francisco Ayarza 281. Urb. Panamericana Norte. Los

Olivos. Lima, Perú.

Teléfono: 947820392

Correo electrónico: lorena.lozano87@hotmail.com

Recibido: 06 de agosto de 2018.

Evaluado: 16 de agosto de 2018

Aprobado: 17 de setiembre de 2018 .

(c) La revista. Publicado por Universidad de San Martín de Porres, Perú. (cc) Br Licencia de Creative Commons Artículo en acceso abierto bajo términos de Licencia Creative Commons Atribución 4.0 Internacional. (http://creativecommons.org/licenses/by/4.0/)

ORCID iDs

Lorena Lozano Villafuerte Luzmila Troncoso Corzo Víctor Noriega Ruiz https://orcid.org/0000-0001-6549-4775 https://orcid.org/0000-0003-1075-874X https://orcid.org/0000-0003-4709-1328 